\title{
The accuracy of MRI in the diagnosis of anterior cruciate ligament injury
}

\author{
Mengou Zhao ${ }^{1}$, Ying Zhou ${ }^{1}$, Junru Chang ${ }^{1}$, Jie Hu ${ }^{1}$, Huixia Liu ${ }^{1}$, Shuhang Wang ${ }^{1}$, Donglei Si ${ }^{1}$, \\ Yaqin Yuan ${ }^{1}$, Haiyan $\mathrm{Li}^{2}$ \\ ${ }^{1}$ Department of Computed Tomography and Magnetic Resonance, Shijiazhuang Third Hospital, Shijiazhuang, China; ${ }^{2}$ Department of Gynaecology, \\ Shijiazhuang Third Hospital, Shijiazhuang, China \\ Contributions: (I) Conception and design: M Zhao, Y Zhou; (II) Administrative support: J Chang, H Li; (III) Provision of study materials or \\ participants: M Zhao, Y Zhou, J Hu, H Liu, S Wang, D Si, Y Yuan; (IV) Collection and assembly of data: All authors; (V) Data analysis and \\ interpretation: M Zhao, J Chang, J Hu, H Liu, S Wang, D Si, Y Yuan, H Li; (VI) Manuscript writing: All authors; (VII) Final approval of manuscript: \\ All authors. \\ Correspondence to: Mengou Zhao. Department of Computed Tomography and Magnetic Resonance, Shijiazhuang Third Hospital, 15 Tiyu Nan \\ Street, Shijiazhuang, China. Email: zmo110975875@126.com.
}

Background: Anterior cruciate ligament (ACL) injury can cause damage to the meniscus and articular cartilage, and may even lead to osteoarthritis. This study aimed to analyze the accuracy and feasibility of magnetic resonance imaging (MRI) imaging signs in the diagnosis of knee joint ACL injury.

Methods: A total of 78 patients admitted to our hospital from June 2018 to October 2019 with highly suspected ACL injury were selected for this prospective study. We used MRI and arthroscopy to diagnose the ACL injuries, and the results of MRI and arthroscopy, as well as the value of MRI in diagnosing ACL injury, were compared.

Results: Among the 78 participants, 66 cases were diagnosed with ACL injury (28 with complete tear, 38 with partial tear), and 12 were normal according to arthroscopy. Among the 66 cases with ACL injury, 63 (95.45\%) were confirmed by MRI detection. The sensitivity, specificity, and accuracy of MRI in the diagnosis of ACL injury were $95.45 \%$ (63/66), 91.67\%, and $94.87 \%$, respectively. The accuracy of MRI in the diagnosis of complete and partial tears were $92.86 \%$ and $94.74 \%$, respectively. Among the four direct MRI signs of ACL injury, the differences in interruption of ACL continuity, thickening and edema, and abnormal walking were statistically significant $(\mathrm{P}<0.05)$. Among the 8 indirect MRI signs, all showed high specificity and low sensitivity, and which the specificity of posterior cruciate ligament index, Notch sign, meniscus exposure sign of posterior ankle, and lateral collateral ligament monolayer display showed higher specificity $(\mathrm{P}<0.05)$.

Conclusions: Examination with MRI offers high accuracy in the diagnosis of ACL injury, and has good consistency with arthroscopic diagnosis, which can provide reliable guidance for the selection and formulation of clinical surgery plans, and might be used as the first choice for the non-traumatic diagnosis of ACL injury.

Keywords: Anterior cruciate ligament injury; magnetic resonance imaging (MRI); arthroscopy; knee joint

Submitted Oct 18, 2020. Accepted for publication Dec 04, 2020.

doi: $10.21037 / \mathrm{atm}-20-7391$

View this article at: http://dx.doi.org/10.21037/atm-20-7391

\section{Introduction}

The anterior and posterior cruciate ligaments are important parts of the knee joint, and have key roles in maintaining the stability of the knee joint (1). Anterior cruciate ligament (ACL) injury is caused by a violent forward impact on the upper back of the calf, and posterior cruciate ligament injury 
is caused by a violent backward impact on the anterior chamber of the upper leg. Clinically, ACL injuries are more commonly seen $(2,3)$. Injury to the ACL can cause damage to the meniscus and articular cartilage, which in turn leads to osteoarthritis (4). The predominant cause of ACL injury is a sudden change in the direction of movement, which causes partial fiber bundle damage. Arthroscopy is a traditional diagnostic method used to identify ACL damage, and has a high sensitivity, but is an invasive examination (5). Recently, MRI examination has become a very effective noninvasive examination method for the diagnosis of ACL injury in clinic, and it can also display other lesions in the joint and determine the location and extent of ligament tear (6). Therefore, in this study, we selected 78 patients with highly suspected ACL injuries as the research participants, analyzed the accuracy of MRI in diagnosing ACL injuries, and compared it with arthroscopy to evaluate the clinical value of MRI in diagnosing ACL injuries. We present the following article in accordance with the STARD reporting checklist (available at http://dx.doi.org/10.21037/atm-207391).

\section{Methods}

\section{General information}

A prospective study was conducted on 78 patients admitted to our hospital from June 2018 to October 2019 who were highly suspected of ACL injury. Among the 78 patients, 55 were male and 23 were female, aged 21-59 years old, with an average age of $42.64 \pm 6.57$ years old. Reasons for treatment included car accidents $(n=7)$, hard object collisions $(n=18)$, sports injuries $(n=37)$, and sprains $(n=16)$. The presenting clinical symptoms included knee swelling, slow movement, and restricted knee movement.

Participants were recruited to this study if they fulfilled the following inclusion criteria: (I) undertaking of both MRI and arthroscopy examination, and received treatment in our hospital; (II) no contraindications to MRI examination; (III) a clear history of trauma; (IV) complete imaging and clinical data; (V) no history of ipsilateral knee surgery.

Patients were excluded from this study if the following criteria were met: (I) obvious signs of osteoarthritis; (II) cochlear implantation; (III) a pacemaker, defibrillator, insulin pump, or indwelling catheter installed in the body; (IV) meniscus disease or developmental deformity (V) women who were either pregnant or lactating. This study was approved by the Shijiazhuang Third Hospital
(No. 2020-10-15). All procedures performed in this study involving human participants were in accordance with the Declaration of Helsinki (as revised in 2013). And the informed consent was taken from all the patients.

\section{MRI examination}

The MRI examination was performed using a Philips Achieva 1.5T magnetic resonance scanner (Philips, Amsterdam, Netherlands). A knee joint flexible coil was selected to detect within a scanning range of $10 \mathrm{~cm}$ between the upper and lower gaps of the knee joint. The scanning sequence parameters was as follows: for protondensity-weighted imaging with fat saturation (PDWI-FS) sagittal position, a radiofrequency (RF) pulse repetition time (TR) of 3,000 ms, echo time (TE) of $31 \mathrm{~ms}$, layer thickness of $4.0 \mathrm{~mm}$, layer spacing of $0.8 \mathrm{~mm}$, and field of view (FOV) of $180 \mathrm{~mm} \times 320 \mathrm{~mm}$ were applied; for PDWI-FS coronal position, TR/TE was $3,000 \mathrm{~ms} / 31 \mathrm{~ms}$, layer thickness of $4.0 \mathrm{~mm}$, layer spacing of $0.8 \mathrm{~mm}$, and FOV of $180 \mathrm{~mm} \times 320 \mathrm{~mm}$; for T1WI sagittal position, TR/TE was $400 \mathrm{~ms} / 31 \mathrm{~ms}$, layer thickness of $4.0 \mathrm{~mm}$, layer spacing of $0.8 \mathrm{~mm}$, and FOV of $180 \mathrm{~mm} \times 320 \mathrm{~mm}$; for T2WI-FS horizontal axis position and fat suppression, TR/TE was $3,770 \mathrm{~ms} / 79 \mathrm{~ms}$, layer thickness of $5 \mathrm{~mm}$, layer spacing of $1.0 \mathrm{~mm}$, and FOV of $180 \mathrm{~mm} \times 384 \mathrm{~mm}$. For 3D-MEDIC (three-dimensional multi-echo combined imaging sequence), TR/TE was $40 \mathrm{~ms} / 22 \mathrm{~ms}$, with a layer thickness of $1.5 \mathrm{~mm}$, and FOV of $150 \mathrm{~mm} \times 384 \mathrm{~mm}$. After the completion of the scan, image post-processing was performed, and the image of participants with ACL injury was reconstructed.

\section{Artbroscopy}

Arthroscopy was performed a week after MRI detection. The participant took the supine position, and after epidural anesthesia, an incision was made on the outside of the knee joint, and the tibial tubercle was incised in a straight line. During the procedure, the ACL was carefully observed, focusing on the area of the lesion shown by the MRI scan, and the probe was used to explore the area of suspected injury to avoid missed diagnosis.

\section{Observation indicators}

The MRI images were interpreted by two physicians using a double-blind method, including $\geq 1$ senior attending 
Table 1 Detection of ACL injury by MRI [n (\%)]

\begin{tabular}{lccc}
\hline \multirow{2}{*}{ MRI detection } & \multicolumn{2}{c}{ Arthroscopy detection } & \multirow{2}{*}{ Total } \\
\cline { 2 - 3 } & Positive & Negative & \\
\hline Positive & $63(95.45)$ & $1(8.33)$ & $64(82.05)$ \\
Negative & $3(4.55)$ & $11(91.67)$ & $14(17.95)$ \\
Total & $66(84.62)$ & $12(15.38)$ & 78 \\
\hline
\end{tabular}

$\mathrm{ACL}$, anterior cruciate ligament; $\mathrm{MRI}$, magnetic resonance imaging.

Table 2 Comparison of MRI and arthroscopy [n (\%)]

\begin{tabular}{lcccc}
\hline \multirow{2}{*}{ MRI detection } & \multicolumn{3}{c}{ Arthroscopy detection } & \multirow{2}{*}{ Total } \\
\cline { 2 - 4 } & \multicolumn{1}{c}{ Complete tear } & Partial tear & Normal & \\
\hline Complete tear & $26(92.86)$ & $2(5.26)$ & $0(0.00)$ & $28(42.42)$ \\
Partial tear & $1(3.57)$ & $36(94.74)$ & $0(0.00)$ & $37(56.06)$ \\
Normal & $1(3.57)$ & $0(0.00)$ & $0(0.00)$ & $1(1.52)$ \\
Total & $28(42.42)$ & $38(57.58)$ & $0(0.00)$ & 66 \\
\hline
\end{tabular}

MRI, magnetic resonance imaging.

physician. In the event of differing opinions, a consensus was achieved by discussion. Using arthroscopy results as the "gold standard" of the diagnosis of ACL injury, the sensitivity, specificity, and accuracy of MRI in diagnosing ACL injury were analyzed.

\section{Statistical methods}

The data in this study were statistically analyzed using SPSS 18.0 software (IBM Corp., Armonk, NY, USA). The measurement data were described as the mean \pm standard deviation $(\bar{x} \pm \mathrm{SD})$; the count data were expressed as a rate or composition ratio, and analyzed using the chi-squared $\left(\chi^{2}\right)$ test. Results with $\mathrm{P}<0.05$ were considered to be statistically significant.

\section{Results}

\section{The sensitivity, specificity and accuracy of MRI in the} diagnosis of ACL injury

Among 78 patients, 66 cases were diagnosed with ACL injury by arthroscopy detection, and the remaining 12 cases were identified as normal. Among the ACL injuries, 28 were complete tear, and 38 were partial tear. There were 63 cases diagnosed as ACL injury by MRI, which gave a detection rate of $95.45 \%$. The sensitivity, specificity, and accuracy of MRI in the diagnosis of ACL injury were 95.45\% (63/66), $91.67 \%$, and $94.87 \%$, respectively (Table 1).

\section{Comparison of the diagnosis results of MRI and artbroscopy}

By comparing the results of MRI and arthroscopy, it was found that the accuracy rate of MRI in the diagnosis of complete tear was $92.86 \%$, and was $94.74 \%$ for partial tear (Table 2).

\section{Assessment results of direct MRI signs of ACL injury}

Among the four direct MRI signs of ACL injury, the differences in interruption of ACL continuity, thickening and edema, and abnormal walking were statistically significant $(\mathrm{P}<0.05$, Table 3).

\section{Assessment results of indirect MRI signs of ACL injury}

Among the eight indirect MRI signs of ACL injury, all of them had high specificity and low sensitivity. The indirect MRI signs of posterior cruciate ligament index, Notch sign, meniscus exposure sign of posterior ankle, and lateral collateral ligament monolayer display showed higher specificity $(\mathrm{P}<0.05$, Table 4$)$.

\section{Image analysis}

After the MRI scan, patients with ACL injury and complete tear showed no continuity of ACL fibers and severed end contracture. There was obvious diffuse thickening and widening at the severed segment, and high signal at the T2WI position (Figure 1). A large amount of effusion signal could be seen in the joint cavity, with low signal at the T1WI position and high signal at T2WI position. In patients with partial tears, the ACL tear was partially swollen, and most of it was not obvious, with the T2WI signal higher than previously (Figure 2).

\section{Discussion}

The ACL originates at the front of the inter-ankle carina of the tibia, slants backward, outward, and upward, and terminates at the medial and posterior sides of the lateral femoral malleolus. The functions of the ACL include 
Table 3 Evaluation results of direct MRI signs of ACL injuries

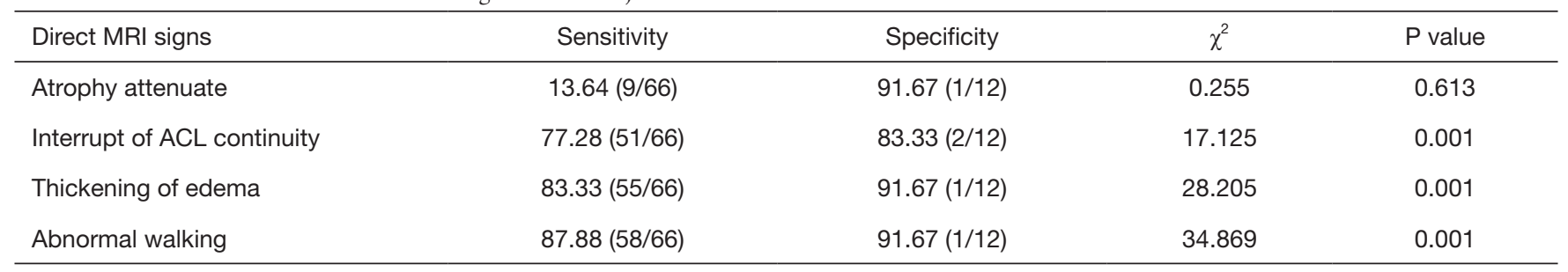

$\mathrm{ACL}$, anterior cruciate ligament; $\mathrm{MRI}$, magnetic resonance imaging.

Table 4 Evaluation results of indirect MRI signs of ACL injuries

\begin{tabular}{lccc}
\hline Indirect MRI signs & Sensitivity & Specificity & $\chi^{2}$ \\
\hline Posterior cruciate ligament angle & $53.03(35 / 66)$ & $91.67(1 / 12)$ & 8.163 \\
Posterior cruciate ligament index & $56.06(37 / 66)$ & $100.00(0 / 12)$ & 12.798 \\
Bone contusion & $54.55(36 / 66)$ & $83.33(2 / 12)$ & 5.831 \\
Notch sign & $36.36(24 / 66)$ & $100.00(0 / 12)$ & 6.001 \\
Anterior tibial displacement & $51.52(34 / 66)$ & $83.33(2 / 12)$ & 4.962 \\
Meniscus exposure sign of posterior ankle & $27.27(18 / 66)$ & $100.00(0 / 12)$ & 0.012 \\
Hollow ankle socket sign & $46.97(31 / 66)$ & $91.67(1 / 12)$ & 0.026 \\
Lateral collateral ligament monolayer display & $25.76(17 / 66)$ & $100.00(0 / 12)$ & 0.039 \\
\hline
\end{tabular}

$\mathrm{ACL}$, anterior cruciate ligament; $\mathrm{MRI}$, magnetic resonance imaging.
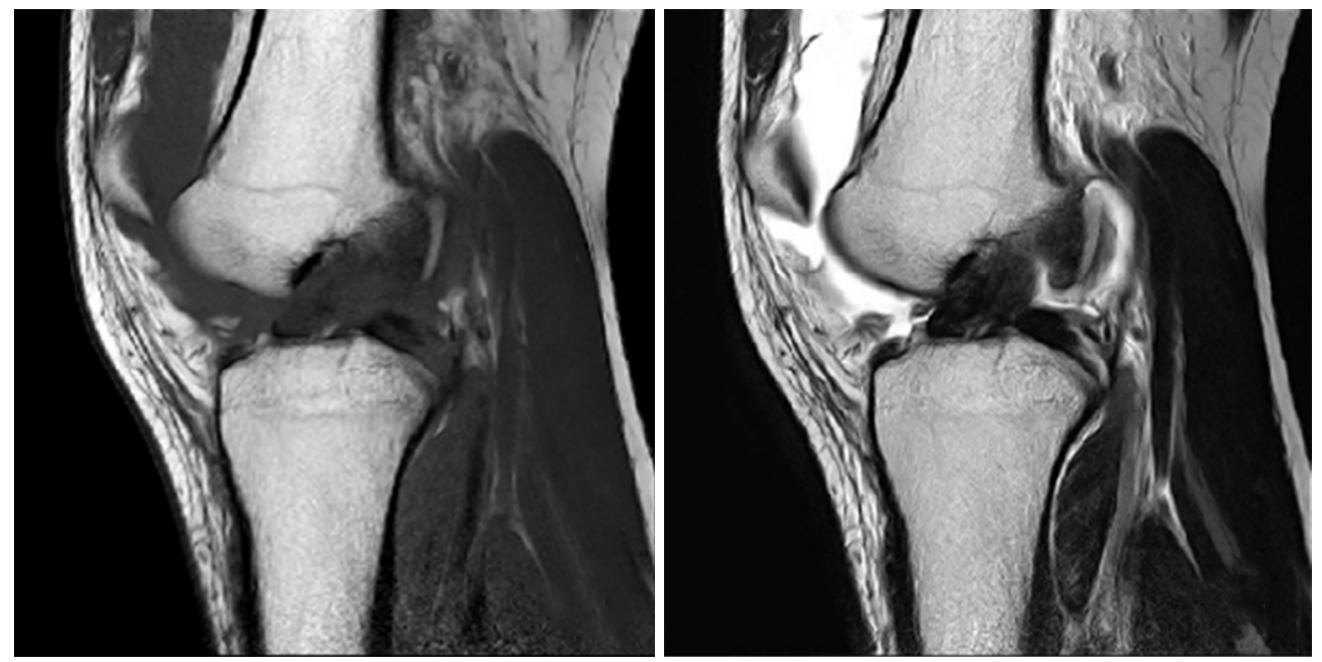

Figure 1 ACL rupture by MRI scanning in sagittal positions T1 and T2. ACL, anterior cruciate ligament; MRI, magnetic resonance imaging.

restricting excessive forward movement of the tibia, hyperextension of the knee joint, rotation of the tibia, and knee extension $(7,8)$. The ACL is at a special position in the knee joint, with highest vulnerability of all its parts. Once the ACL is damaged, it is difficult for the body to repair by itself. If the injury is not treated in time, it can cause tearing and degeneration of the knee joint meniscus, soft tissue, and other organs, eventually leading to osteoarthritis (9). 

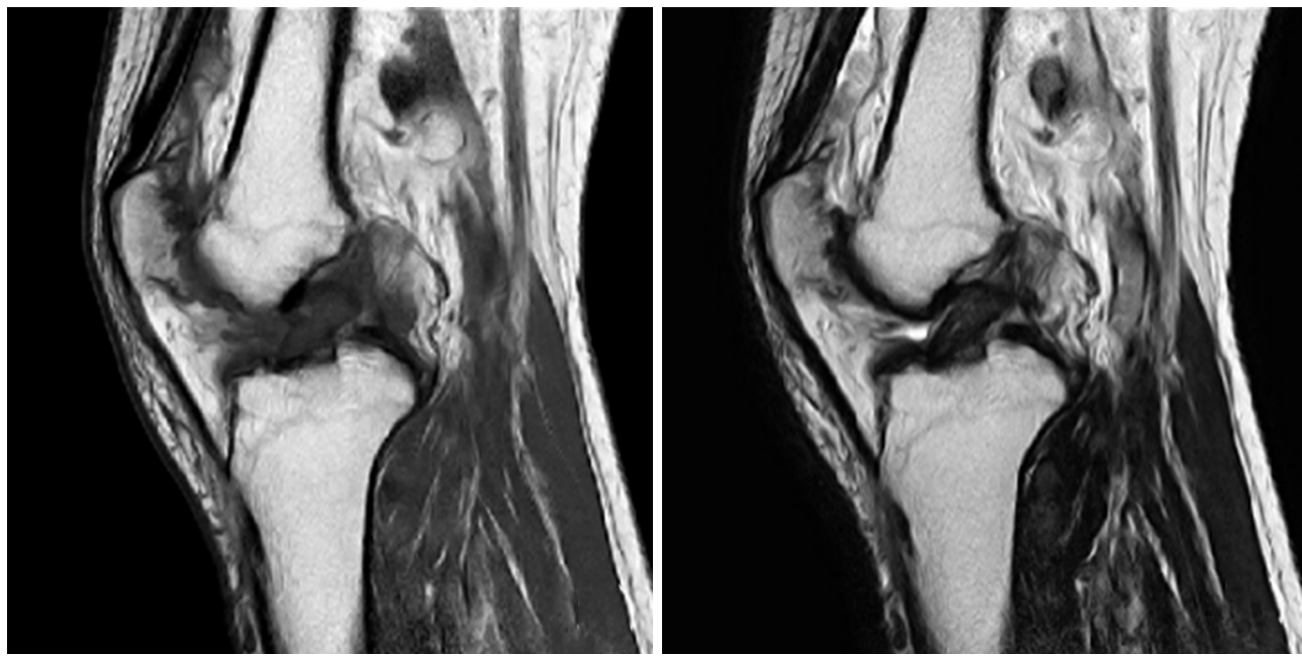

Figure 2 Partial injury in ACL by MRI scanning in sagittal positions T1 and T2. ACL, anterior cruciate ligament; MRI, magnetic resonance imaging.

With unique characteristics and properties, ACL injuries of the knee joint are classified into contact and non-contact injuries according to whether an impact was involved. In addition, Sobue et al. (10) have classified ACL injury into simple ACL injury, ACL with medial structure injury, ACL with lateral structure injury, and ACL with medial and lateral structure injury. Injuries to the ACL mostly occur in the middle of the ligament, accounting for about $70 \%$, followed by the 7 points of the femoral lateral malleolus ( 9-20\%), and then finally the tibial insertion $(<10 \%)(11,12)$. Following ACL injury, patients will experience swelling of the knee joint, pain, and dysfunction, which impact seriously on their personal and work lives. Therefore, accurate diagnosis of ACL injury is of great significance to patients.

Examination with MRI is a kind of tomography, and also a kind of emission tomography; it can be imaged without radioisotope injection, which stands it apart from positron emission tomography (PET) and single-photon emission computerized tomography (SPECT) (13). There are many advantages to MRI, such as high spatial resolution, high soft tissue resolution, which can clearly display the overall structure of the knee joint, and facilitate clinical observation of the patient's anterior and posterior cruciate ligament injuries (14). The hydrogen atoms in normal ACLs are fixed on the net frame composed of polypeptides and do not participate in MR imaging. Therefore, ACLs always exhibit banded low signals about $1 \mathrm{~cm}$ in width in each sequence (15). When injury occurs, the most intuitive manifestation is the change of ACL signal and the ability to walk. Due to the destruction of the peptide network structure and the accumulation of liquid, the ligament swells with diffused high signal, works abnormally, and may break, twist, or even disappear (16). The stability of the knee joint is destroyed with ACL injury, and the tibia will move forward, resulting in abnormalities in the shape and position of the meniscus and posterior cruciate ligament. These abnormalities can be used as indirect signs for the diagnosis of ACL injury $(17,18)$.

In this study, a total of 63 cases were detected with ACL injury by MRI among the 66 ACL injury patients detected by arthroscopy. Among the direct signs, there were 5 with high specificity. Among the 5, the signs of interruption of ACL continuity, thickening and edema, and abnormal walking continuity of the ACL were more sensitive to diagnosis than other signs, and the most common sign was abnormal gait following the onset of ACL injury. Among the indirect signs, 8 showed high specificity with low sensitivity, indicating that all of the 8 indirect signs have certain diagnostic values. Although indirect signs cannot be used as a direct diagnostic basis for the determination of ACL injury, they can be used as an auxiliary basis for the diagnosis of ACL injury. Therefore, the combination of direct and indirect signs can effectively improve the accuracy of ACL injury diagnosis.

The arthroscope is a rod-shaped optical instrument with a diameter of $\sim 5 \mathrm{~cm}$ to observe the internal structure of joints; it is an endoscope for the diagnosis and treatment of joint diseases (19). The internal structure of the joint can be directly observed through arthroscopy, and arthroscopy is 
the current "gold standard" for the diagnosis of ACL injury, with an accuracy rate as high as $100 \%$ (20). However, arthroscopy is invasive and presents certain risks to patients. Therefore, many patients are unwilling to accept this method when seeking diagnosis. The biggest difference between MRI examination and arthroscopy is that MRI is a non-invasive examination, has high resolution, high sensitivity, and high specificity for ACL injury diagnosis. The results of this study showed that the sensitivity of MRI in diagnosing ACL damage was $95.45 \%$, and the specificity was $91.67 \%$. Additionally, this study used MRI to diagnose ACL tears in patients with ACL injuries. The results showed that compared with arthroscopic detection, the accuracy of MRI in diagnosing complete and partial ACL tears was as high as $90 \%$, indicating that the efficacies of MRI examination and arthroscopic investigation in diagnosing ACL injury are similar, and that the diagnostic results are consistent between the two methods. However, misdiagnosis by MRI examinations was present in this study, and may have been caused by hemorrhage and fluid accumulation around the ligaments following acute trauma. In addition, different scanning angles can also result in misdiagnosis.

In summary, diagnosis of ACL injury using MRI has high accuracy and good consistency with arthroscopic diagnosis. It can provide reliable guidance for the selection and formulation of clinical surgery plans, and can be used as the first choice for the non-invasive diagnosis of ACL injury.

\section{Acknowledgments}

Funding: None.

\section{Footnote}

Reporting Checklist: The authors have completed the STARD reporting checklist. Available at http://dx.doi.org/10.21037/ atm-20-7391

Data Sharing Statement: Available at http://dx.doi. org/10.21037/atm-20-7391

Conflicts of Interest: All authors have completed the ICMJE uniform disclosure form (available at http://dx.doi. org/10.21037/atm-20-7391). The authors have no conflicts of interest to declare.

Ethical Statement: The authors are accountable for all aspects of the work in ensuring that questions related to the accuracy or integrity of any part of the work are appropriately investigated and resolved. This study was approved by the Shijiazhuang Third Hospital (No. 2020$10-15)$. All procedures performed in this study involving human participants were in accordance with the Declaration of Helsinki (as revised in 2013). And the informed consent was taken from all the patients.

Open Access Statement: This is an Open Access article distributed in accordance with the Creative Commons Attribution-NonCommercial-NoDerivs 4.0 International License (CC BY-NC-ND 4.0), which permits the noncommercial replication and distribution of the article with the strict proviso that no changes or edits are made and the original work is properly cited (including links to both the formal publication through the relevant DOI and the license). See: https://creativecommons.org/licenses/by-nc-nd/4.0/.

\section{References}

1. Samuelsson K, Magnussen RA, Alentorn-Geli E, et al. Equivalent Knee Injury and Osteoarthritis Outcome Scores 12 and 24 Months After Anterior Cruciate Ligament Reconstruction: Results from the Swedish National Knee Ligament Register. Am J Sports Med 2017;45:2085-91.

2. Eijgenraam SM, Reijman M, Bierma-Zeinstra SMA, et al. Can we predict the clinical outcome of arthroscopic partial meniscectomy? A systematic review. Br J Sports Med 2018;52:514-21.

3. Wright $M$, Chesterton $\mathrm{P}$, Wijnbergen $M$, et al. The Effect of a Simulated Soccer Match on Anterior Cruciate Ligament Injury Risk Factors. Int J Sports Med 2017;38:620-6.

4. Bouras T, Fennema P, Burke S, et al. Stenotic intercondylar notch type is correlated with anterior cruciate ligament injury in female patients using magnetic resonance imaging. Knee Surg Sports Traumatol Arthrosc 2018;26:1252-7.

5. Williams A, Winalski CS, Chu CR. Early articular cartilage MRI T2 changes after anterior cruciate ligament reconstruction correlate with later changes in $\mathrm{T} 2$ and cartilage thickness. J Orthop Res 2017;35:699-706.

6. Nagelli CV, Cook JL, Kuroki K, et al. Does Anterior Cruciate Ligament Innervation Matter for Joint Function and Development of Osteoarthritis? J Knee Surg 2017;30:364-71.

7. Sánchez-Carrasco MA, Abellán JF, Qudsi-Sinclair S, et 
al. Comparison of Outcomes of Two Femoral Fixation Devices in Hamstring Anterior Cruciate Ligament Reconstruction. Indian J Orthop 2017;51:487-92.

8. Shen L, Jin ZG, Dong QR, et al. Anatomical Risk Factors of Anterior Cruciate Ligament Injury. Chin Med J (Engl) 2018;131:2960-7.

9. Lie MM, Risberg MA, Storheim K, et al. What's the rate of knee osteoarthritis 10 years after anterior cruciate ligament injury? An updated systematic review. Br J Sports Med 2019;53:1162-7.

10. Sobue Y, Kojima T, Kurokouchi K, et al. Prediction of progression of damage to articular cartilage 2 years after anterior cruciate ligament reconstruction: use of aggrecan and type II collagen biomarkers in a retrospective observational study. Arthritis Res Ther 2017;19:265.

11. Saltzman BM, Meyer MA, Weber AE, et al. Prospective clinical and radiographic outcomes after concomitant anterior cruciate ligament reconstruction and meniscal allograft transplantation at a mean 5-year follow-up. Am J Sports Med 2017;45:550-62.

12. Gandolfi M, Ricci M, Sambugaro E, et al. Changes in the sensorimotor system and semitendinosus muscle morphometry after arthroscopic anterior cruciate ligament reconstruction: a prospective cohort study with 1-year follow-up. Knee Surg Sports Traumatol Arthrosc 2018;26:3770-9.

13. Roemer FW, Englund M, Turkiewicz A, et al. Molecular and structural biomarkers of inflammation at two years after acute anterior cruciate ligament injury do not predict structural knee osteoarthritis at five years. Arthritis Rheumatol 2019;71:238-43.

Cite this article as: Zhao M, Zhou Y, Chang J, Hu J, Liu H, Wang S, Si D, Yuan Y, Li H. The accuracy of MRI in the diagnosis of anterior cruciate ligament injury. Ann Transl Med 2020;8(24):1657. doi: 10.21037/atm-20-7391
14. Inokuchi T, Matsumoto $\mathrm{T}$, Takayama $\mathrm{K}$, et al. Influence of the Injury-to-Surgery Interval on the Healing Potential of Human Anterior Cruciate Ligament-Derived Cells. Am J Sports Med 2017;45:1359-69.

15. Nagai T, Bates NA, Hewett TE, et al. Effects of localized vibration on knee joint position sense in individuals with anterior cruciate ligament reconstruction. Clin Biomech (Bristol, Avon) 2018;55:40-4.

16. Jin C, Song EK, Jin QH, et al. Outcomes of simultaneous high tibial osteotomy and anterior cruciate ligament reconstruction in anterior cruciate ligament deficient knee with osteoarthritis. BMC Musculoskelet Disord 2018;19:228.

17. Arumugam A, Strong A, Tengman E, et al. Psychometric properties of knee proprioception tests targeting healthy individuals and those with anterior cruciate ligament injury managed with or without reconstruction: a systematic review protocol. BMJ Open 2019;9:e027241.

18. Tutkus V, Tutkus J, Jablonskiene V, et al. Tibial part of anterior cruciate ligament: a host for silent foreign body in the knee. Chin Med J (Engl) 2018;131:1884-6.

19. Harput G, Tok D, Ulusoy B, et al. Translation and crosscultural adaptation of the anterior cruciate ligament-return to sport after injury (ACL-RSI) scale into Turkish. Knee Surg Sports Traumatol Arthrosc 2017;25:159-64.

20. van Tol FR, Kernkamp WA, van der Wal RJP, et al. The occurrence of meniscal and chondral injury in two-stage revision anterior cruciate ligament reconstruction: a consecutive case series. J Knee Surg 2020;33:223-7.

(English Language Editor: J. Jones) 